\title{
artigo
}

\section{O papel do profissional de odontologia no atendimento de manifestações bucais em pacientes com leucemia}

\author{
The role of the dentistry professional in care of oral manifestations in patients with leukemia
}

El papel del profesional de odontología en el cuidado de las manifestaciones orales en pacientes con leucemia

\begin{abstract}
RESUMO
Objetivo: revisar artigos disponíveis em periódicos científicos que exemplificassem o papel do profissional de odontologia no atendimento de manifestações bucais em pacientes com Leucemia. Metódo: tratou-se de uma revisão bibliográfica narrativa utilizando artigos selecionados datados dos anos de 2008 a 2018. Resultados: As manifestações bucais das leucemias são mais frequentes nas formas agudas, do que nas formas crônicas. 0 profissional da saúde deve estar sempre atento a qualquer tipo de sinal ou sintoma, assim como à constante atualização da história médica. Com o surgimento de novas técnicas que propiciem a manutenção da saúde bucal nos pacientes com algum tipo de neoplasia, hoje é possível prevenir uma doença sem que ela já esteja instalada. Conclusão: Conclui-se que a presença do profissional de odontologia na equipe oncológica pode diminuir a morbidade e a mortalidade relacionadas a complicações bucais, assim como aumentar o conforto e qualidade de vida dos pacientes durante a terapia.
\end{abstract}

DESCRITORES: Odontologia; Leucemia; Manifestações bucais.

\section{ABSTRACT}

Objective: to review articles available in scientific journals that exemplify the role of the dental professional in the care of oral manifestations in patients with Leukemia. Method: it was a narrative bibliographic review using selected articles from the years 2008 to 2018. Results: Oral manifestations of leukemia are more frequent in acute forms, than in chronic forms. The health professional must always be attentive to any type of sign or symptom, as well as the constant updating of medical history. With the emergence of new techniques that promote the maintenance of oral health in patients with some type of neoplasia, today it is possible to prevent a disease without it being installed. Conclusion: It is concluded that the presence of the dental professional in the oncology team can reduce the morbidity and mortality related to oral complications, as well as increase the comfort and quality of life of patients during therapy.

DESCRIPTORS: Dentistry; Leukemia; Oral manifestations.

\section{RESUMEN}

Objetivo: revisar artículos disponibles en revistas científicas que ejemplifiquen el papel del profesional odontológico en el cuidado de las manifestaciones bucales en pacientes con leucemia. Método: se realizó una revisión bibliográfica narrativa utilizando artículos seleccionados de los años 2008 a 2018. Resultados: Las manifestaciones orales de leucemia son más frecuentes en las formas agudas que en las crónicas. El profesional de la salud debe estar siempre atento a cualquier tipo de signo o síntoma, así como a la actualización constante de la historia clínica. Con la aparición de nuevas técnicas que favorecen el mantenimiento de la salud bucal en pacientes con algún tipo de neoplasia, hoy es posible prevenir una enfermedad sin que se instale. Conclusión: Se concluye que la presencia del profesional odontológico en el equipo de oncología puede reducir la morbimortalidad relacionada con las complicaciones bucales, así como incrementar la comodidad y calidad de vida de los pacientes durante la terapia. DESCRIPTORES: Odontología; Leucemia; Manifestaciones orales.

RECEBIDO EM: 30/07/2020 APROVADO EM: 23/09/2020

\section{Josanne Christine Araújo Silva}

Bacharel em Nutrição pelo Centro Universitário de Ciências e Tecnologia do Maranhão - UniFacema; Pós-Graduada em Nutrição Clínica, Funcional e Fitoterapia pelo Centro Universitário de Ciências e Tecnologia do Maranhão - UniFacema; Pós-graduada em Gestão e Docência do Ensino Superior pela Faculdade Seven - FAEME.

ORCID: 0000-0003-3181-8406 


\section{Erick Michell Bezerra Oliveira}

Bacharel em Fisioterapia na UNIFACEMA-2019. Acadêmico do curso de Odontologia na UNIFACEMA. Pós-graduando em Docência do Ensino Superior com ênfase em sistema de saúde-FAVENI. Pós-Graduando em Gestão Hospitalar e Auditoria em Serviços de Saúde na Estratego Sistema Educacional.

ORCID: 0000-0001-6056-2863

\section{Hernandys Ribeiro Bezerra}

Acadêmico do curso de Odontologia no Centro Universitário de Ciências e Tecnologia do Maranhão-UNIFACEMA. ORCID: 0000-0001-7261-3920

\section{Flávio Bruno Rodrigues de Assunção}

Acadêmico do curso de Odontologia no Centro Universitário de Ciências e Tecnologia do Maranhão-UNIFACEMA. ORCID: 0000-0001-5982-7542

\section{Josienne Araújo da Silva}

Acadêmico do curso de Odontologia no Centro Universitário de Ciências e Tecnologia do Maranhão-UNIFACEMA. ORCID: 0000-0002-1551-1941

\section{Emerson de Sousa Pinheiro}

Acadêmico do curso de Odontologia no Instituto Florence de Ensino Superior- IFES. ORCID: 0000-0003-3844-470X

\section{INTRODUÇÃO}

A Leucemia é um grupo de doenças malignas, complexas e diferentes entre si, caracterizadas pela produção excessiva e progressiva de leucócitos, que surgem no sangue em formas imaturas, a luz da literatura. Essa doença afeta cerca de $13 \mathrm{em}$ 100 mil pessoas todos os anos, sendo o tipo linfocítico crônico o mais comum. Na leucemia, o crescimento de leucócitos é desordenado e independente, sendo que estes perdem suas funções, com frequência levando o paciente ao óbito. A etiologia da maioria das leucemias é incerta, mas alguns autores citam infecção viral, exposição à radiação ionizante e outros tipos de radiação eletromagnética, além de exposição química. ${ }^{1,2}$

Ainda em relação á Leucemia, existem complicações orais resultantes da terapia do câncer que acometem alguns pacientes, mas que podem ser reduzidas quando algumas medidas no âmbito da odontologia são estabelecidas para equilibrar a cavidade oral antes do início da quimioterapia minimizando os efeitos que esta provoca e também em possíveis intercorrências sistêmicas, de acordo com a literatura. Com isso, o principal objetivo do odontólogo é promover um tratamento odontológico onde haja o equilíbrio e a prevenção das complicações da quimioterapia. ${ }^{3}$
Medidas preventivas como instrução sobre higiene oral e detecção precoce das lesões orais, são altamente eficazes quando estabelecidas no início do tratamento. $\mathrm{O}$ exame oral permite ao cirurgião dentista determinar as condições bucais e estabelecer as intervenções necessárias para reduzir as complicações durante e após a terapia. Para que ocorra sucesso do tratamento, o médico responsável deve comunicar ao cirurgião dentista sobre o estado de saúde do paciente e o plano de tratamento instituído para o mesmo e ao mesmo tempo deve ser informado quais as medidas devem ser tomadas para os cuidados orais antes, durante e após a quimioterapia. ${ }^{1,4}$

Em concordância com a literatura, o cirurgião dentista possui papel fundamental no auxílio do diagnóstico precoce e na intervenção de melhoria da saúde bucal destes pacientes. $\mathrm{Na}$ quimioterapia surgem as mucosites, a xerostomia e a imunodepressão, causando infecções dentárias e oportunistas. Esta terapia possui efeito antitumoral que destrói ou retarda a divisão das células com proliferação acelerada, assim como as células tumorais. Portanto, este tratamento não diferencia as células neoplásicas das células normais com alta atividade mitótica como é o caso das células da mucosa oral, tornando esta região susceptível ao aparecimento de lesões pela dificuldade de renovação celular causada pelas drogas. ${ }^{5,6,7}$

O protocolo de atendimento pretende destacar os cuidados necessários a serem tomados, bem como as medidas que devem necessárias nas complicações causadas pela quimioterapia. Estes pacientes apresentam a imunidade comprometida e por esse motivo precisam de cuidados mais efetivos para a manutenção de sua saúde oral. ${ }^{5}$

Tendo em vista que estes pacientes apresentam a imunidade comprometida e por esse motivo precisam de cuidados mais efetivos para a manutenção de sua saúde oral, faz-se o questionamento sobre o papel do profissional odontológico junto á esses pacientes, questão esta que norteou a criação deste estudo. Nota-se o valor dos procedimentos odontológicos para o atendimento das manifestações orais da leucemia, apesar de ser um tema muito debatido, faz se necessário à criação de novos estudos com intuito de disponibilizar informações importantes para estes pacientes. Para isso, o objetivo deste estudo foi revisar artigos disponíveis em periódicos científicos que exemplificassem o papel do profissional de odontologia no atendimento de manifestações bucais em pacientes com Leucemia. 


\section{MÉTODOS}

Este estudo constitui uma revisão bibliográfica narrativa de caráter descritivo a respeito do papel do profissional de odontologia no atendimento de manifestações bucais em pacientes com Leucemia. A coleta de dados foi realizada entre os intervalos de 2008 á 2018, e utilizou-se para as pesquisas as bases de dados Biblioteca Digital Brasileira de Teses e Dissertações (BDTD), Coordenação de Aperfeiçoamento de Pessoal de Nível Superior (CAPES) e Google Acadêmico. A análise para seleção dos estudos foi realizada em duas fases. $\mathrm{Na}$ primeira fase, os artigos foram selecionados segundo os critérios de inclusão e exclusão. Como busca geral na base de dados BDTD foram encontrados na sua totalidade duzentos e quarenta e sete (147) estudos, ao filtrar os mesmos, disponíveis em sua totalidade, publicados entre os anos de 2008 á 2018, nos idiomas português e inglês, obteve-se um total de cento e quarenta e três (43) publicações. Nas bases de dados Google Acadêmico obteve-se oito mil, trezentos e sessenta (360) artigos, ao aplicar os filtros textos completos gratuitos obteve-se cinco mil cento e oitenta (180) artigos. Na base de dados CAPES, obteve-se um total de três mil duzentos e sessenta e sete (267) artigos na busca geral, ao aplicar o filtro de artigos publicados nos últimos dez anos obteve-se quarenta e um (41) artigos.

$\mathrm{Na}$ segunda fase, foram analisados de forma independente os títulos e os resumos dos artigos, para verificar se os mesmos estavam condizentes com a temática abordada no presente estudo, sendo observados ainda dados como: nome do autor ou autores, ano de realização, objetivos, metodologia do estudo, resultados e discussão. Ao final, foram selecionados para a análise sete (7) artigos na base de dados BDTD, cinco (5) artigos na base de dados Google Acadêmico e três (3) artigos na base de dados CAPES, totalizando 15 trabalhos. Por fim, foram seguidos os seguintes passos: leitura exploratória, leitura seletiva e escolha do material que contemplasse os objetivos deste estudo, análise dos textos e, por último, a realização de leitura interpretativa e redação.

Na leucemia aguda, a cavidade bucal

está sujeita às

complicações que

variam desde lesões

de mucosa e

infecções, até

a exacerbação

de condições

patológicas

preexistentes.

\section{RESULTADOS E DISCUSSÃO}

Os estudos selecionados nesta revisão analisaram o papel do profissional de odontologia no atendimento de manifestações bucais em pacientes com leucemia. As leucemias são neoplasias hematológicas malignas e heterogêneas, que têm a sua origem em células da medula óssea. De acordo com o estudo de Carneiro, Silva e Cruz $(2008)^{6}$, os primeiros casos foram relatados no século XIX com a observação de alteração da medula óssea em pacientes que foram a óbito. Para Lowal e colaboradores $(2015)^{8}$, a hematopoese consiste no processo de maturação em cascata da linhagem hematopoiética, iniciada pelas células pluripotentes que residem na medula óssea. A leucemia decorre de um erro genético que compromete esse processo de maturação. Dependendo do tipo da linha- gem comprometida, a leucemia é classificada como mieloide ou linfoide. A forma linfoide ou linfoblástica pode ser dividida segundo as células afetadas em: de células $B$ ou de células T. ${ }^{8}$

De acordo com Menezes e Rao $(2012)^{10}$, as manifestações bucais das leucemias são mais frequentes nas formas agudas, do que nas formas crônicas. Os achados podem surgir como primeiros sinais e sintomas da doença. Sua rápida detecção conduz ao precoce diagnóstico e tratamento, melhorando as chances do paciente atingir a remissão e reduzindo a frequência e severidade das complicações.

Dessa maneira, a anamnese completa se caracteriza como a etapa mais importante para a promoção de saúde bucal. Costa, Silva e Macedo (2011) ${ }^{13}$, retratam em sua publicação que o profissional da saúde deve estar sempre atento a qualquer tipo de sinal ou sintoma, assim como à constante atualização da história médica. Na leucemia aguda, a cavidade bucal está sujeita às complicações que variam desde lesões de mucosa e infecções, até a exacerbação de condições patológicas preexistentes. ${ }^{11,12}$

Infecçôes sistêmicas podem surgir a partir de complicações bucais, colocando em risco a vida do paciente. Entretanto, as complicações bucais causam, com frequência, severo desconforto, o que pode interferir na nutrição e atrapalhar a conclusão da terapia. Deliverksa e Krasteva (2013) afirmam que o comprometimento da cavidade bucal pode surgir no estágio inicial da leucemia, aumentando sua intensidade, com a indução de mielossupressão pela terapia antileucêmica, tendendo a regredir com a remissão da doença. A frequência e o tipo de sinais e sintomas encontrados no momento do diagnóstico variam muito de indivíduo para indivíduo e dependem do tipo de leucemia., ${ }^{5,13}$

Os autores Moucherek e Trovão $(2010)^{7}$ descrevem em seu artigo que entre as manifestações estomatológicas iniciais das leucemias agudas frequentemente observadas estão: sangramento, presença de petéquias e equimoses, úlceras, hiperplasia gengival e palidez da mucosa. $\mathrm{O}$ número de pacientes, com Leucemia, que procuraram primeiramente o dentista têm 
aumentado constantemente, assim, esse profissional tem sido o responsável pelos primeiros passos para se chegar ao diagnóstico de leucemia. As lesões primárias são resultantes da infiltração direta dos tecidos bucais pelas células leucêmicas. Zimmermann e colaboradores $(2015)^{11}$ retratam que as lesões secundárias estão associadas com a anemia, a trombocitopenia e a granulocitopenia, sendo o resultado da invasão da medula óssea e substituição das células saudáveis pelas células neoplásicas. As lesões terciárias estão relacionadas com a terapia adotada e incluem efeitos de citotoxicidade direta e indireta.

O estudo de Hespanhol e colaboradores $(2010)^{12}$ alega que os pacientes com câncer fazem parte do universo dos pacientes com necessidades especiais e como tal necessitam de um atendimento odontológico diferenciado, não só para cuidados rotineiros com a cavidade bucal, mas por implicações causadas à mesma pelo próprio tratamento a que são submetidas. Esses pacientes necessitam de rigorosos cuidados especiais quanto ao tratamento odontológico, principalmente devido às complicações consequentes dos efeitos colaterais da quimioterapia. Fica evidenciado no estudo de Zerbini (2011) ${ }^{14}$ que é fundamental intervir nas complicações bucais da quimioterapia, pois quando estão presentes e dependendo da sua gravidade, podem comprometer o tratamento médico, afetando desde a nutrição do paciente quando a recuperação do mesmo.

Em referência á pacientes oncológicos, Reenesh, Munishwar e Rath (2012) ${ }^{15}$, relatam que estes devem ser examinados pelo cirurgião-dentista tão logo tenham sua doença diagnosticada. $\mathrm{O}$ tratamento deve ser iniciado preferencialmente antes do tratamento oncológico. Esses pacientes necessitam de prioridade no atendimento odontológico para evitar o retardo do tratamento antineoplásico.

As orientações dadas previamente aos pais sobre o controle das condições bucais contribuem para reduzir o índice de complicações orais decorrentes do tratamento antineoplásico. Arul e colaboradores $(2012)^{16}$ destacam a aplicação da quimioterapia e a da radioterapia tem sido muito efetiva, porém estão associadas a efeitos colaterais significantes, incluindo toxicidade aos tecidos hematopoéticos e não-hematopoéticos.

\section{Por outro lado, os}

alimentos ásperos, que podem causar abrasão da mucosa, os muito espessos para a quantidade mínima de saliva, os alimentos muito condimentados, temperados, ou picantes, ou alimentos que irritam ou queimam a mucosa bucal, como os sucos

\section{cítricos e as bebidas}

\section{alcoólicas devem}

\section{ser evitados.}

Segundo Silva e colaboradores $(2012)^{17}$, entre os efeitos colaterais da cavidade bucal clinicamente importante, encontra-se a interrupção das funções e integridade dos tecidos bucais, resultando mucosite, gengivite, candidíase, xerostomia, trismo, cárie, osteorradionecrose, celulite e erupções na mucosa. Presença do cirurgião dentista na equipe multidisciplinar oncológica é de fundamental importância na prevenção das complicações.

Para Grando et al., $(2015)^{18}$ os pacientes devem ser submetidos a uma avaliação dentária completa, incluindo radiografias, antes do início da quimioterapia. Se o estado geral de saúde do paciente permitir, os dentes sem possibilidade de tratamento devem ser extraídos e os demais restaurados, para reduzir a possibilidade de infecção. $\mathrm{O}$ tratamento endodôntico deve ser considerado como alternativa às extrações, nos pacientes que tenham um elevado nível de higiene oral. No entanto, a terapia endodôntica não está indicada em pacientes com granulocitopenia e trombocitopenia.

Pode haver distúrbios nutricionais devido aos efeitos sistêmicos e locais da quimioterapia. Para Caldas Junior et al $(2015)^{19}$,deve-se considerar uma dieta rica em sacarose, carboidratos refinados, alimentos frios e líquidos. Por outro lado, os alimentos ásperos, que podem causar abrasão da mucosa, os muito espessos para a quantidade mínima de saliva, os alimentos muito condimentados, temperados, ou picantes, ou alimentos que irritam ou queimam a mucosa bucal, como os sucos cítricos e as bebidas alcoólicas devem ser evitados. A redução da ingestão de alimentos, de acordo com Gondim e colaboradores $(2010)^{20}$, pode gerar danos como estomatite, dor na boca e debilitação geral. A náusea e os vômitos também prejudicam a ingestão de alimentos e aumentam a perda de proteínas e de vitaminas necessárias à estrutura das células epiteliais.

Com o surgimento de novas técnicas e utilização de meios que propiciem a manutenção da saúde bucal nos pacientes com algum tipo de neoplasia, hoje é possível prevenir uma doença sem que ela já esteja instalada. Por esse motivo é de extrema importância considerar um protocolo de atendimento adequado e minucioso para estes pacientes com o objetivo de melhorar a condição da saúde bucal deles além de melhorar sua qualidade de vida. 


\section{artigo}

Araújo Silva, J.C.; Oliveira, E.M.B.; Assunção, F.B.R.; Araújo da Silva, J.; Pinheiro, E.S.;

O papel do profissional de odontologia no atendimento de manifestações bucais em pacientes com leucemia

\section{CONCLUSÃO}

A leucemia pode ter suas primeiras manifestações na mucosa bucal, o que reafirma a importância do Cirurgião-Dentista no atendimento em relação á doença. Após o diagnóstico, a presença deste profissional na equipe oncológica pode diminuir a morbidade e a mortalidade relacionadas a complicações bucais, assim como aumentar o conforto e a qualidade de vida dos pacientes durante a terapia.

Todas as medidas que foram relata- das exigem conhecimento e habilidades prévias adquiridas pelo profissional. Esta conduta deve-se principalmente ao fato de que indivíduos acometidos por alguma neoplasia têm uma progressão rápida da doença e a não intervenção em curto espaço de tempo pode resultar em danos irreversíveis na qualidade de vida deles.

A partir das informações obtidas podemos concluir que, fazem-se necessários novos estudos sobre o público aqui abordado, permitindo uma avaliação clínica e prática, que demonstre possíveis formas de melhorar o atendimento de manifestações bucais em pacientes com Leucemia.

Assim, sugere-se o incentivo a publicação de mais artigos científicos na área. Faz-se oportuno considerar os trabalhos já descritos na comunidade acadêmica, no intuito de fortalecer pesquisas futuras sobre este tema. Como revela o artigo, são vários os autores que procuram deixar clara a importância do papel do profissional de odontologia no atendimento de manifestações bucais em pacientes com Leucemia.

\section{REFERÊNCIAS}

1. Lobão D, S, Oliveira B, M, Massara M, L, A, Viana M, B, Nunes L. Condições da Cavidade Bucal e Acompanhamento Odontológico de Crianças com Leucemia Linfocítica Aguda. Rev Med Minas Gerais 2008; 18(4 Supl 1): S25-S32. 2008.

2. Brasil. Ministério da Saúde. Instituto Nacional do Câncer José Alencar Gomes da Silva. Tipos de Câncer: leucemia. Estimativas do Câncer 2016.

3. Frazão C, O, B, Alfaia T, A, Costa R, C, Rocha M, L, Gouveia C, V, $D$, Morais A, P. Pacientes oncológicos pediátricos: Manifestações bucais da terapia antineoplásica. Rev saúde e pesquisa 2012; 5(3):559-64.

4. Barbosa A, M, Ribeiro D, M, Caldo Teixeira A, S. Conhecimentos e práticas em saúde bucal com crianças com câncer hospitalizadas. Ciênc saúde coletiva2010; 15(1):1113-22.

5. Salazar M, Victorino F, R, Paranhos I, R, Ricci I, D, Galti W, Caçador N, P. Efeitos do tratamento da radioterapia de cabeça e pescoço de interesse ao cirurgião dentista: Revisão de literatura. Rev odonto. 200816 (31):62-8

6. Carneiro F, M, Silva L, C, P, Cruz R, A. Manifestações bucais das leucemias agudas na infância. Arq bras odontol 2008; 4(1):4054.

7. Moucherek JR. J, C, E, Trovão M, N, A. Avaliação dos índices de placa e gengival de crianças portadoras de neoplasias submetidas a tratamento antineoplásico. Rev brasil odont 2010; 67(1):101-5.

8. Lowal K, A, Alaizari N, A, Tarakji B, Petro W, Hussain K, A, Altamini $M, A, A$. Dental considerations for leukemic pediatric patients: um updated review for general dental practitioner. Mater Sociomed. 2015; 27(5): 359-62.

9. Deliverksa $E, G$, Krasteva $A$. oral signs of leukemia and dental management - literature data and case report. Journal of IMAB Annual Proceeding (Scientific Papers). 2013; (19)4: 388-91.

10. Menezes L, Rao J, R. Acute myelomonocytic leukemia presenting with gingival enlargement as the only clinical manifestation. J Indian Soc Periodontol 2012; 16:597-60.
11. Zimmermann C, Meurer M, I, Grando L, J, Del Moral J, A, G, Rath I, B, S, Tavares S, S. Dental Treatment in Patients with Leukemia. J Oncol. 2015; 2015:571739

12. Hespanhol F, L, Tinoco E, M, B, Teixeira H, G, C, Falabella M, E, V, Assis N, M, S, P. Manifestações bucais em pacientes submetidos à quimioterapia. Ciênc. saúde coletiva. 2010; 15(1): 108594.

13. Costa S, S, Silva A, M, Macedo I, A, B. Conhecimento de manifestações orais da leucemia e protocolo de atendimento odontológico. Rev de Odontol da Univ. Cid de São Paulo. 2011, 23(1):70-8.

14. Zerbini M, C, N, Soares F, A, Morais J, C, Vassallo J, Velloso $E, D, R, P$, Chaufaille $M, L, L$. Classificação dos tumores hematopoéticos e linfoides de acordo com a OMS: padronização da nomenclatura em língua portuguesa, $4^{\mathrm{a}}$ edição. J Bras Patol Med Lab. 2011; 47(6):643-8.

15. Reenesh M, Munishwar S, Rath S, K. Generalised leukaemic gingival enlargement: a case report. J ORAL MAXILLOFAC RES. 2012; 3(3):5.

16. Arul A, S, K, J, Verma S, Ahmed S, Arul A, S, S, J. A clinical and fine needle aspiration cytology study of gingiva in acute leukemia. Dent Res J (Isfahan). 2012; 9(1):80-5.

17. Silva B, A, Siqueira C, Castro P, Araújo S, S, Volpato L. Oral manifestations leading to the diagnosis of acute lymphoblastic leukemia in a young girl. J Indian Soc Pedod Prev Dent. 30:166-8. 2012.

18. Grando L, J, Mello A, L, F, S, Salvato L, Brancher A, P, Del Moral J, A, G, Steffenellodurigon G. Impact of leukemia and lymphoma chemotherapy on oral cavity and quality of life. Spec Care Dentist. 35(5): 236-42. 2015.

19. Caldas Junior A, Barbosa A, Teti I, M, França M, L, M, S, Paegle A, C, R, O, Cauás M. Alterações bucais em pacientes submetidos ao tratamento quimioterápico de câncer na rede pública de Recife-PE Ciênc biol saúde. 2(2): 37-46. 2015.

20. Gondim F, M; Gomes I, P; Firmino F. Prevenção e tratamento da mucosite oral. Rev enferm UERJ. 18(1): 67-74. 2010. 